Chirurg 2020 $91: 905-912$

https://doi.org/10.1007/s00104-020-01242-3

Online publiziert: 24 . Juli 2020

(c) Springer Medizin Verlag GmbH, ein Teil von Springer Nature 2020

Redaktion

U. Settmacher Jena

\section{F. Braun' -A. Rahmel}

'Sektion Klinische Transplantation, Klinik für Allgemeine, Viszeral-, Thorax-, Transplantations- und Kinderchirurgie, Universitätsklinikum Schleswig-Holstein (UKSH), Campus Kiel, Kiel, Deutschland ${ }^{2}$ Deutsche Stiftung Organtransplantation (DSO), Frankfurt am Main, Deutschland

\title{
Änderungen im Transplantationsgesetz und Auswirkungen auf das Spender- aufkommen in Deutschland
}

\begin{abstract}
Die Transplantationsmedizin nahm in Deutschland in den 1960er-Jahren ihren Anfang. Im Jahr 1963 fand hierzulande die erste Nierentransplantation statt. Inzwischen gehören Transplantationen zu den standardisierten Verfahren in der Medizin und ermöglichen für ausgewählte Patienten die häufig einzige effiziente und erfolgreiche Therapie bei irreversiblem Organversagen. Das zentrale Problem ist nach wie vor die limitierte Verfügbarkeit von Spenderorganen.
\end{abstract}

In den letzten 60 Jahren wurden in Deutschland mehr als 139.000 Organe verpflanzt, die schwer kranken Menschen die Chance auf ein neues Leben ermöglichten. Aber immer noch übersteigt die Zahl der Patienten, die von einer Transplantation profitieren könnten, die Zahl der gespendeten Organe.

Im Jahr 2017 hatte der Anteil an Organspendern in Deutschland mit 9,7 Spendern pro 1 Mio. Einwohner einen Tiefstand erreicht, auch im europäischen Vergleich. Der Mangel an Organen und seine vielschichtigen Ursachen haben die Politik zum Handeln aufgerufen.

Im Jahr 1997 trat das deutsche Transplantationsgesetz (TPG) in Kraft [1]. Es regelt die Spende, Entnahme, Vermittlung und Übertragung von Organen, die nach dem Tod oder zu Lebzeiten gespendet werden. Dabei trennt das Gesetz die Bereiche Organspende, Or- ganvermittlung und Organübertragung organisatorisch und personell streng voneinander. Das TPG sieht zudem verschiedene Kontrollmechanismen vor, um Missbrauch zu verhindern, und schafft Rechtssicherheit für Spender und ihre Angehörigen, Empfänger und alle an der Organentnahme und Transplantation Beteiligten. Der Transparenz und Chancengleichheit unter allen Organempfängern wird Sorge getragen, da die Verteilung der gespendeten Organe transparent nach bundesweit einheitlichen Richtlinien erfolgt, die von der Bundesärztekammer (BÄK) nach dem Stand der medizinischen Wissenschaft kontinuierlich weiterentwickelt werden. Die Allokationskriterien berücksichtigen insbesondere Erfolgsaussicht, Dringlichkeit sowie Chancengleichheit.

Gemäß TPG wurde die Deutsche Stiftung Organtransplantation (DSO) als Koordinierungsstelle für die postmortale Organspende damit beauftragt, die Zusammenarbeit der beteiligten Partner im Organspendeprozess zu organisieren. Dazu gehören alle Schritte beginnend mit den notwendigen vorbereitenden Maßnahmen bis zum Transport und der Übergabe der Organe in die Transplantationszentren. Die Stiftung Eurotransplant ist als Vermittlungsstelle zuständig für die Allokation der Spenderorgane, die für Deutschland nach den Richtlinien der BÄK erfolgt. Die Transplantationszentren sind mit der Indikationsstellung, der Führung der Wartelisten, der Durchfüh- rung der Transplantation, der Nachsorge und der Qualitätssicherung beauftragt.

\section{》) Jährlich sterben in \\ Deutschland etwa 1000 Pati- enten, während sie auf eine Transplantation warten}

Technische Innovationen wie Split- oder Dominotransplantation, Verwendung von marginalen Spenderorganen sowie Maschinenperfusion können den Bedarf an Spenderorganen allerdings bis heute nicht kompensieren, sodass Patienten weiterhin lange Wartezeiten für eine Transplantation erdulden müssen. Alternativen wie Xenotransplantation oder der Einsatz artifizieller Organe haben die klinische Einsatzreife noch nicht erreicht. Ende 2019 standen in Deutschland rund 9000 schwer kranke Patienten auf den Wartelisten für ein Organ. Diese Zahl unterschätzt die Anzahl derjenigen, die von einer Transplantation profitieren könnten, betrachtet man allein schon die mehr als 90.000 hiesigen Dialysepatienten. Jährlich sterben in Deutschland etwa 1000 Patienten, während sie auf eine Transplantation warten.

\section{Analyse der Ursachen des Organspendemangels}

Eine erste Analyse des Organspendepotenzials in deutschen Krankenhäusern erfolgte mit dem Pilotprojekt zur 
Inhousekoordination bei Organspenden, an dem bundesweit 112 Kliniken zwischen Anfang 2010 bis Mitte 2012 teilnahmen [2]. Initiator war die DSO, unterstützt vom Deutschen Krankenhausinstitut (DKI). Die Entnahmehäuser wurden in die Kategorien Universitätskliniken (A), Krankenhäuser mit Neurochirurgie (B) und Krankenhäuser ohne Neurochirurgie (C) unterteilt.

Kernelemente der Inhousekoordination waren eine Standardisierung und Dokumentation aller Abläufe um die Organspende: von der Früherkennung möglicher Spender bis zur Entnahme von Organen. Dazu gehörte auch eine gemeinsame Analyse von Mitarbeitern der Klinik (Inhousekoordinatoren) und der DSO (DSO-Koordinatoren) zum Spenderpotenzial. Basis dafür sind die anonymisierten Todesfälle nach primärer und sekundärer Hirnschädigung. Sowohl die A-Kliniken als auch andere teilnehmende Kliniken wiesen eine konstante Spenderrate auf, wohingegen B-Kliniken und nicht teilnehmende Kliniken eine eher rückläufige Spenderrate hatten. Als Ursachen wurden eine mangelnde Spendererkennung, der aufwendige Organspendeprozess sowie die wachsende Zahl an Therapielimitierungen und Patientenverfügungen diskutiert, die eine mögliche Organspende bereits im Vorfeld vermeintlich ausschließen. Die identifizierten strukturellen Defizite sollten durch die Einführung von Transplantationsbeauftragten (ehemals Inhousekoordinatoren) behoben werden. Im Rahmen der TPG-Novellierung 2012 wurden diese in den Entnahmekrankenhäusern etabliert. Die Ernennung zum Transplantationsbeauftragten $(\mathrm{TxB})$ wurde jedoch damals häufig als Zusatztätigkeit und nicht als Karrierechance verstanden, sodass es zu keiner spürbaren Strukturverbesserung in der Organspende kam.

Die 2018 publizierten Studienergebnisse einer Kieler Arbeitsgruppe wiesen abermals auf ein strukturelles Problem in den Krankenhäusern hin [3]. In die bundesweite Untersuchung flossen mehr als 100 Mio. vollstationäre Behandlungsfälle aus den Jahren 2010 bis 2015 ein. Von diesen wurden diejenigen Patienten mit schwerer Hirnschädigung identifiziert, die grundsätzlich für eine Or- ganspende infrage gekommen wären. Die Zahl der tatsächlich realisierten Organspenden lag jedoch deutlich niedriger. Als Ursache für diese Diskrepanz sahen die Forscher Defizite beim Erkennen und Melden von möglichen Spendern in den Entnahmekrankenhäusern. Diese könnten wiederum durch eine hohe Arbeitsverdichtung aufden Intensivstationen sowie die Komplexität und auch Seltenheit einer Organspende bedingt sein. Gelänge es, diesen Prozess in den Krankenhäusern organisatorisch und politisch zu stärken, könnte die Anzahl der gespendeten Organe erheblich gesteigert werden, so das Fazit der Studie aus Kiel.

In der Zeitschrift Der Anästhesist wurde im Januar 2019 eine noch detailliertere Analyse zu nicht erfolgten Spendermeldungen veröffentlich [4]. Die Autoren haben mithilfe des Computerprogramms „DSO-TransplantCheck für Excel“ für das Jahr 2016 alle Todesfälle von Patienten mit primärer und sekundärer Hirnschädigung in Entnahmekrankenhäusern aus der DSO-Region Ost (Sachsen, Sachsen-Anhalt, Thüringen) identifiziert. Bei den Verstorbenen aus dieser Gruppe wurden die Fälle genauer untersucht, in denen keine Diagnostik des irreversiblen Hirnfunktionsausfalls (IHA) durchgeführt wurde. Auf diesem Weg konnten weitere mögliche Organspender identifiziert werden, bei denen

- eine indizierte IHA-Diagnostik nicht durchgeführt wurde,

- eine Patientenverfügung vermeintlich keine Fortführung der Therapie gestattete,

- die Therapie aufgrund einer infausten Prognose limitiert wurde, ohne dass die Option einer Organspende mit den Angehörigen besprochen wurde.

Aus den erhobenen Daten wurden Empfehlungen abgeleitet, um sicherzustellen, dass die Möglichkeit einer Organspende in diesen Fällen immer bedacht wird:

- Auch wenn eine Patientenverfügung bei infauster Prognose vermeintlich eine weitere Intensivtherapie ausschließt, sollte vor Therapieabbruch der Patientenwille bzw. mutmaßliche Patientenwille bezüglich einer Organspende evaluiert werden.
- Ein Therapieabbruch bei infauster Prognose ohne Evaluierung des (mutmaßlichen) Patientenwillens bezüglich einer Organspende entspricht weder dem Gedanken des TPG noch dem Anspruch an die Patientenautonomie.

- Das Hinzuziehen von TxB und Neurointensivmedizinern hilft in der Beurteilung des klinischen Verlaufes und der weiteren Behandlungsplanung bei schwer hirngeschädigten Patienten.

In der Bevölkerung sind die Zustimmungsraten zur Organspende über die letzten Jahre weitgehend konstant geblieben. Laut einer 2018 durchgeführten Umfrage der Bundeszentrale für gesundheitliche Aufklärung (BZgA) standen $84 \%$ der befragten Bundesbürger der Organspende positiv gegenüber. Gleichzeitig ist der Anteil derjenigen, die laut der Umfrage angeben, einen Organspendeausweis zu besitzen, von $17 \%$ im Jahr 2008 auf $36 \%$ im Jahr 2018 angestiegen [5].

》) Nur 15\% der für Organspende
infrage kommenden Verstorbe-
nen hatten ihre Entscheidung
schriftlich festgehalten

Betrachtet man die tatsächlichen Organspender, so zeigt sich, dass nach wie vor in vielen Fällen die Angehörigen eine Entscheidung gemäß dem bekannten oder vermuteten Willen des Verstorbenen treffen. DSO-Daten für 2019 ergaben, dass nur $15 \%$ der für eine Organspende infrage kommenden Verstorbenen ihre Entscheidung dazu schriftlich festgehalten hatten. Mehr als $40 \%$ der Ablehnungen wurden von Angehörigen getroffen, die oft gar nicht wissen, was der Verstorbene wollte, und die sich daher eher gegen eine Organspende entscheiden [6].

Die schriftliche oder mündliche $\mathrm{Zu}$ stimmung zur Organspende befindet sich allerdings mitunter im Widerspruch zu einer vorhandenen Patientenverfügung. Im Vordergrund steht häufig die frühzeitige Begrenzung intensivmedizinischer 
Hier steht eine Anzeige.

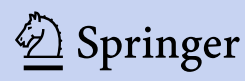


Maßnahmen ohne das Thema Organspende, das eine zumindest zeitlich befristete Fortführung der intensivmedizinischen Maßnahmen erfordert, anzusprechen. Um den Patientenwillen in dieser für Angehörige und intensivmedizinisches Personal herausfordernden Situation umsetzen zu können und gleichzeitig für alle Beteiligten Rechtssicherheit zu schaffen, hat die BÄK in einem Arbeitspapier Formulierungen vorgeschlagen, die es ermöglichen, in der Patientenverfügung einer möglichen Organspende zeitlich befristet den Vorrang vor dem unmittelbaren Abbruch der intensivmedizinischen Maßnahmen einzuräumen.

\section{Maßnahmen der Politik zur Verbesserung der Organspendesituation}

Die Politik hat mit Weitblick gehandelt, indem sie die strukturellen Probleme in der Organspende von der Diskussion um die rechtliche Regelung der Entscheidung zur Organspendebereitschaft getrennt und durch unterschiedliche Gesetzesnovellierungen geregelt hat.

Zum 01.04.2019 trat das „Zweite Gesetz zur Änderung des Transplantationsgesetzes - Verbesserung der Zusammenarbeit und der Strukturen bei der Organspende" in Kraft [7]. Die damit veränderten Rahmenbedingungen sollen insbesondere den Kliniken ihre Aufgaben im Organspendeprozess erleichtern und gleichzeitig durch eine flächendeckende Berichtspflicht für mehr Verbindlichkeit und Transparenz in der Erkennung möglicher Spender sorgen. Im Fokus der Maßnahmen stehen eine Stärkung der $\mathrm{TxB}$ und der Abläufe in den Kliniken sowie eine aufwandsgerechtere Finanzierung.

Die wesentlichen Punkte dieses Gesetzes sind:

1. verbindliche Freistellung der $\mathrm{TxB}$ und deren Finanzierung,

2. höhere Vergütung der Entnahmekrankenhäuser,

3. weitere Maßnahmen zur Verbesserung des Prozessablaufs in der Organspende:

- Stärkung der allgemeinen Stellung des TxB im Entnahmekrankenhaus,

Chirurg 2020 -91:905-912 https://doi.org/10.1007/s00104-020-01242-3

(c) Springer Medizin Verlag GmbH, ein Teil von Springer Nature 2020

\section{F. Braun · A. Rahmel}

\section{Änderungen im Transplantationsgesetz und Auswirkungen auf das Spenderaufkommen in Deutschland}

\section{Zusammenfassung}

Seit Jahrzehnten besteht in Deutschland ein Mangel an Spenderorganen, 2017 erreichte die Zahl der Organspender einen historischen Tiefstand. Eine sorgfältige Analyse der Ursachen zeigte u. a. strukturelle Defizite in der Spendererkennung und -meldung und veranlasste die politisch Verantwortlichen zum Handeln. Am 01.04.2019 trat das Gesetz zur Verbesserung der Zusammenarbeit und der Strukturen in der Organspende in Kraft, das insbesondere die Rolle der Transplantationsbeauftragten in den Entnahmekrankenhäusern stärkt und für eine aufwandsgerechte Finanzierung der organspendebezogenen Aufgaben der Entnahmekrankenhäuser sorgt. Gleichzeitig wird die Kooperation der Transplantationsbeauftragten mit der Koordinierungsstelle gefördert. Um die Auseinandersetzung mit dem Thema Organspende in der Bevölkerung zu fördern, folgte am 16.01.2020 das Gesetz über die Stärkung der Entscheidungsbereitschaft bei der Organspende. Die Umsetzung der verschiedenen Einzelmaßnahmen der beiden Gesetze wird von einem Gemeinschaftlichen Initiativplan Organspende begleitet. Die rechtlichen Bestimmungen und deren praktische Umsetzung werden ausführlich dargestellt.

Schlüsselwörter

Organspende - Spenderorganmangel . Transplantationsbeauftragte · Initiativplan . Spendererkennung

\section{Amendments to the Transplantation Act and impact on the donor situation in Germany}

\section{Abstract}

In Germany, the scarcity of donor organs has persisted over decades and reached an historical low point in the year 2017. A thorough analysis of the causes revealed structural deficits in the identification and registration of possible donors as one of the central reasons for the low donation rate. This prompted the political authorities to act and resulted in two new laws, which led to a modification of the German Transplantation Act. On 1 April 2019, the Act on Improvement of the Cooperation and the Structural Framework for Organ Donation came into force. This Act strengthens the role of the transplant coordinators in the harvesting hospitals and establishes adequate reimbursement of the organ donationrelated costs in the harvesting hospitals. Furthermore, it fosters the cooperation of the transplant coordinators with the German organ procurement organization. On 16 January 2020, the existing opt-in legislation was modified. While the general principle of the opt-in legislation stayed unchanged, different measures were introduced that aim to repeatedly inform all citizens about organ donation and thereby motivate them to make a decision on organ donation. In order to enable a reliable and transparent documentation an organ donor registry will be established. The practical implementation of the various measures of both Acts is supported by a multi-institutional collaborative initiative plan for organ donation. The legal regulations and their practical implementation are depicted in detail.

\section{Keywords}

Organ donation - Donor organ scarcity . Transplant coordinator · Initiative plan · Donor recognition
- Maßnahmen zur Verbesserung des Organspendeprozesses in den Kliniken,

- flächendeckende Bereitstellung eines neurochirurgischen und neurologischen konsiliarärztlichen Rufbereitschaftsdienstes,
- Stärkung des Unterstützungsangebots für die Entnahmekrankenhäuser und für die $\mathrm{TxB}$,

- Einrichtung einer Qualitätssicherung in den Entnahmekrankenhäusern, 
4. rechtliche Grundlage für die Angehörigenbetreuung,

5. Verfahrensvereinfachungen.

Um die gesetzlichen Rahmenbedingungen in die Praxis zu überführen, wurde mit mehreren Partnern ein Gemeinschaftlicher Initiativplan Organspende erarbeitet und im Juni 2019 der Öffentlichkeit vorgestellt [8]. Die federführende Rolle - mit Unterstützung des Bundesgesundheitsministeriums - oblag dabei der DSO. Drei wesentliche Handlungsfelder stehen dabei im Vordergrund:

- Das erste Handlungsfeld betrifft Prozesse vor Feststellung des irreversiblen Hirnfunktionsausfalls und Behandlungsstrategien am Lebensende - wie das Denken an die Organspende bei Patienten mit schwerer Hirnschädigung. Hier sollen primär die TxB bei der praktischen Umsetzung ihrer Aufgaben unterstützt werden, z. B. mit Schulungen, beim Umgang mit Patientenverfügungen oder beim Informationsaustausch und der Vernetzung in den Kliniken bzw. Intensivstationen sowie untereinander.

- Ein zweites Handlungsfeld bezieht sich auf die Prozesse nach Feststellung des irreversiblen Hirnfunktionsausfalls sowie auf die Spendermeldung und -charakterisierung, d.h. auf Maßnahmen, die die entsprechenden Abläufe in den Kliniken unterstützen und optimieren.

- Das dritte Handlungsfeld betrifft die stärkere gesellschaftliche Verankerung und Wertschätzung der Organspende in der Öffentlichkeit. Das impliziert die Einbindung von Multiplikatoren und eine differenziertere Aufklärungsarbeit nach Zielgruppen.

Ausgehend von diesen Handlungsfeldern wurden 12 Empfehlungen zur Förderung der Organspende entwickelt, die sich an die Transplantationsbeauftragten, die Entnahmekrankenhäuser, die Multiplikatoren in Medizin und Gesellschaft sowie an die Öffentlichkeit richten.

Nach Regelung der strukturellen Verbesserungsmaßnahmen stand nach Jahren die Entscheidungslösung auf dem
Prüfstand. Bei der Abstimmung über die Entscheidung zur Organspende setzte sich der Antrag für eine Beibehaltung einer Zustimmungslösung gegenüber der Einführung einer Widerspruchslösung durch. Das Gesetz über die Stärkung der Entscheidungsbereitschaft bei der Organspende wurde am 16.01.2020 verabschiedet [9]. Es wird voraussichtlich im ersten Quartal 2022 in Kraft treten.

Das neue Gesetz sieht unter anderem vor:

- Die Einrichtung eines bundesweiten Onlineregisters beim Bundesinstitut für Arzneimittel und Medizinprodukte (BfArM).

- Die Ausweisstellen von Bund und Ländern müssen den Bürgerinnen und Bürgern zukünftig Aufklärungsmaterial und Organspendeausweise aushändigen bzw. bei elektronischer Antragstellung elektronisch übermitteln. Dabei wird auf weitere Informations- und Beratungsmöglichkeiten sowie die Möglichkeit, sich vor Ort oder später in das Onlineregister einzutragen, hingewiesen.

- Hausärztinnen und Hausärzte können künftig bei Bedarf ihre Patientinnen und Patienten alle 2 Jahre über die Organ- und Gewebespende ergebnisoffen beraten. Das Gesetz sieht außerdem vor, die Organ- und Gewebespende verstärkt in der ärztlichen Ausbildung zu verankern.

- Grundwissen zur Organspende soll zudem in den Erste-Hilfe-Kursen im Vorfeld des Erwerbs der Fahrerlaubnis vermittelt werden.

\section{Umsetzung der gesetzlichen Maßnahmen in der Praxis}

\section{Finanzierung und Freistellung der Transplantationsbeauftragten}

Das Gesetz zur Verbesserung der Zusammenarbeit und der Strukturen bei der Organspende sieht für die TxB eine Mindestfreistellung von 0,1 Stellenanteilen einer Vollzeitkraft (VK) pro 10 Intensivstationsbetten für die Behandlung eines möglichen Organspenders vor. Transplantationszentren müssen mindestens eine volle Stelle für die Aufgaben des $\mathrm{TxB}$ einrichten. Falls der
Mindestaufwand überschritten wird, ist der Mehraufwand zu begründen. Die Freistellung der inzwischen rund $1600 \mathrm{TxB}$ wird den Krankenhäusern vergütet (2020 $13.000 €$ je 0,1 VK), die Umsetzung der Vergütung erfolgt durch die DSO. Voraussetzung sind eine namentliche Nennung der TxB gegenüber der DSO, die unterschriebene Vereinbarung über die erfolgte TxB-Freistellung und die Dokumentation der Tätigkeit der TxB. Der Tätigkeitsnachweis erfolgt durch die Berichterstattung des $\mathrm{TxB}$ und des Entnahmekrankenhauses, den Nachweis von internen und externen Fortbildungen sowie die Erstellung und Aktualisierung von krankenhausinternen Verfahrensanweisungen (SOP). Der TxB-Bericht enthält die Analyse des hausinternen Spenderpotenzials auf der Basis eines definierten Datensatzes, der alle Todesfälle des Krankenhauses mit Diagnosen, Beatmungszeitraum und Abteilungszugehörigkeit beinhaltet. Das Computerprogramm TransplantCheck 4 ist im Internet über die Website der DSO verfügbar und erleichtert diese Analyse. Todesfälle, die kein klares Ausschlusskriterium gegen eine Organspende aufweisen, müssen anhand einer Einzelfallanalyse aufgearbeitet werden, sodass mögliche, nicht realisierte Organspender identifiziert und quantifiziert werden können. Die prozessuale Einzelfallanalyse erlaubt zudem Rückschlüsse, in welcher Phase der Organspendeprozess aufgehalten wurde.

Das Gesetz stattet den TxB mit uneingeschränktem Zugang zu den Intensivstationen und Krankenunterlagen aus. Idealerweise sollten dem jährlich verpflichtenden Berichtswesen eine Prozessanalyse und Strukturoptimierung folgen. Zur ihrer strukturellen und inhaltlichen Unterstützung hat die BÄK zudem eine „AG Transplantationsbeauftragte“ eingerichtet.

\section{Finanzierung der Aufgaben der Entnahmekrankenhäuser im Ablauf einer Organspende}

Zum 01.04.2019 wurden durch die Änderung des TPG die Vergütungsregelungen für die Abgeltung der Leistungen in den Entnahmekrankenhäusern mit dem 
Ziel neu gestaltet, die organspendebezogenen Strukturen in den Entnahmekrankenhäusern zu verbessern und diese dem tatsächlichen Aufwand entsprechend angemessen zu vergüten. Dies gilt insbesondere für die Einführung eines Zuschlages als Ausgleich dafür, dass ihre Infrastruktur im Rahmen einer Organspende in besonderem Maße in Anspruch genommen wird.

Die Pauschale setzt sich wie folgt zusammen:

- Grundpauschale IHA-Diagnostik (A),

- Grundpauschale ICU(,intensive care unit")-Therapie (B),

- Grundpauschale OP(Operation)Phase (C),

- Infrastrukturzuschlag $[(\mathrm{D})=2 \times(\mathrm{A}+$ $\mathrm{B}+\mathrm{C})]$.

Aus den Pauschalen A-D ergibt sich der Gesamtbetrag $[(E)=(A+B+C)+D]$. Die Pauschale (A) für die IHA-Diagnostik wird ohne Beteiligung von Konsilärzten, die einen Honoraranspruch gegenüber der DSO haben, mit $1300 €$ und mit Inanspruchnahme eines Konsilarztes mit $800 €$ vergütet. Wurde die IHADiagnostik ausschließlich von Konsilärzten, die ihren Einsatz gegenüber der DSO in Rechnung stellen, durchgeführt, kann das Krankenhaus keine IHA-Grundpauschale geltend machen. Die differenzierte Vergütung soll den Anteil hausinterner IHA-Diagnostik stärken. Die finanzielle Vergütung einer realisierten Multiorganentnahme mit einer ausschließlich durch diensthabende Ärzte des Krankenhauses durchgeführten IHA-Diagnostik bildet sich somit folgendermaßen für das Jahr 2020 ab:

- Grundpauschale IHA-Diagnostik (A): $1300 €$,

- Grundpauschale ICU-Therapie (B): $1494 €$,

- Grundpauschale OP-Phase (C): 3998€,

- Zwischensumme (A + B + C): $6792 €$,

- Infrastrukturzuschlag $[(\mathrm{D})=2 \times(\mathrm{A}+$ B + C)]: $13.584 €$,

- Gesamtbetrag $[(\mathrm{E})=(\mathrm{A}+\mathrm{B}+\mathrm{C})+$ D]: $20.376 €$.

Die Pauschalen werden jährlich neu durch das INEK (Institut für das Ent- geltsystem im Krankenhaus gGmbH) kalkuliert und von der Deutschen Krankenhausgesellschaft (DKG) veröffentlicht.

\section{Neurokonsiliarischer Bereitschafts- dienst}

Der neurokonsiliarische Bereitschaftsdienst soll 24/7 eine flächendeckende Verfügbarkeit qualifizierten Personals für die Feststellung des IHA sicherstellen. Zur Organisation dieses Rufbereitschaftsdienstes beauftragen der Spitzenverband Bund der Krankenkassen, die BÄK und die DKG eine geeignete Einrichtung. Diese darf weder an der Entnahme noch an der Übertragung der Organe beteiligt sein.

\section{Höhere Wertschätzung der Organ-} spender und ihrer Angehörigen

Der Initiativplan soll u.a. die stärkere gesellschaftliche Verankerung und Wertschätzung der Organspende in der Öffentlichkeit fördern, z. B. als bundesweite Veranstaltung zur Würdigung der Organspender. Die erste dieser Art fand am 30.09.2019 im Park des Dankens, des Erinnerns und des Hoffens in Halle (Saale) statt. Dort kamen Angehörige von Organspendern, Organempfänger, Wartelistenpatienten, Mediziner und Politiker zusammen, um der verstorbenen Organspendern zu gedenken. Mit diesem überregionalen Treffen, das nun jährlich stattfindet, wurde der symbolische Grundstein für eine größere gesellschaftliche Anerkennung der Organspende gelegt.

\section{Nächste Schritte und kritische Wertung}

\section{Zweites Gesetz zur Änderung des TPG}

Die Umsetzung der Freistellung stellt nicht selten eine Herausforderung für die Krankenhäuser dar. Das Gros der Entnahmekrankenhäuser hat weniger als 30 relevante Intensivbetten, was einem freizustellenden Stellenanteil von 0,1 bis 0,3 VK entspricht. Hierbei handelt es ich um hoch qualifizierte und spezialisierte Ärzte, die gerade in kleineren Kranken- häusern durch eine Aufstockung des Personals nur schwer kurzfristig kompensatorisch entlastet werden können. Insbesondere in der Übergangsphase unterliegen die TxB daher einer Doppelbelastung, da Einstellungsverfahren und Einarbeitungszeiten überbrückt werden müssen.

In Krankenhäusern der Maximalversorgung erscheint die Implementierung von zusätzlichen pflegerischen $\mathrm{TxB}$ eine wirtschaftliche attraktive Lösung, da die pauschale Vergütung für ärztliche und pflegerische TxB gegenwärtig nicht differenziert wird.

Die Steuerung der budgetären Umsetzung obliegt der Klinikleitung, wobei in größeren Kliniken mitunter zwischen verschiedenen Partikularinteressen $\mathrm{zu}$ vermitteln ist. Eine mögliche Lösung ist dabei die Einrichtung einer übergeordneten Stabsstelle, die beim Vorstand angesiedelt ist, und die Freigabe der finanziellen Mittel steuert und prüft. Eine offene Rückkopplung zwischen dieser Stabsstelle und den TxB ist unerlässlich, damit diese ihren Tätigkeiten in vollem Umfang nachgehen können. Die Überprüfung der zweckgebundenen Mittelverwendung obliegt krankenhausintern der Krankenhausleitung sowie übergeordnet der DSO, damit diese nicht zweckentfremdet verwendet werden.

Die Ministerien sind föderal angehalten, die Organspendeaktivitäten in den Entnahmekrankenhäusern zu überprüfen. Dazu ist gesetzlich vorgesehen, dass sie die Daten zur Todesfallanalyse in den einzelnen Krankenhäusern von der DSO regelmäßig erhalten. Nun gilt es, Erfahrungen mit diesem Vorgehen zu sammeln und ein Maßnahmenkonzept zu entwickeln, das bundeseinheitlich strukturiert ist und gleichzeitig regionale Besonderheiten berücksichtigt.

Insbesondere in kleineren Krankenhäusern ist die Organspende häufig ein seltenes Ereignis und stellt somit mitunter eine besondere Herausforderung dar. Die mittlere Zahl an Organspendern pro Entnahmekrankenhaus lag im Jahr 2017 in den $35 \mathrm{~A}-\mathrm{KH}$ (Krankenhaus) bei 7,5, in den $122 \mathrm{~B}-\mathrm{KH}$ bei 2,83 und in den $1097 \mathrm{C}-\mathrm{KH}$ bei 0,17 . Während in allen A-KH Organspenden realisiert werden konnten, erfolgte in 33 der $123 \mathrm{~B}-\mathrm{KH}$ 
und in 937 der 1091 C-KH keine Organspende. Durch die geplante flächendeckende Bereitstellung eines neurochirurgischen und neurologischen konsiliarärztlichen Rufbereitschaftsdienstes wird die Durchführung der IHA-Diagnostik flächendeckend zeitnah mit hoher Expertise auch in Krankenhäusern ohne die eigene notwendige Erfahrung auf diesem Gebiet gewährleistet. Aber auch andere Aspekte der Betreuung von Organspendern erfordern spezielle Expertise. Eine enge Kooperation eines Krankenhauses der Maximalversorgung mit umliegenden kleineren assoziierten Häusern als Satelliten im Sinne eines Plateausystems könnte sich zur Unterstützung der Prozesse günstig auswirken und die kleineren Kliniken in ihrer Tätigkeit stärken, sodass die Organspende nachhaltig gefördert wird.

\section{Gesetz zur Stärkung der Entscheidungsbereitschaft}

Eine besondere Herausforderung in der Praxis ist die nach wie vor häufig fehlende Dokumentation der Entscheidung zur Organspende. In Umfragen geben ca. $40 \%$ der Bundesbürger an, einen Organspendeausweis zu besitzen. Nach den Erfahrungen der DSO liegt bei den an die DSO berichteten Spendern allerdings nur bei knapp $15 \%$ eine schriftliche Dokumentation vor. Noch ernüchternder sind die Daten einer kürzlich im Deutschen Ärzteblatt publizierten Studie des Universitätsklinikums Essen [10]: Nur knapp 1\% der 2044 unfallchirurgischen Schockraumpatienten, die zwischen Februar 2017 und März 2019 behandelt wurden, führte einen Organspendeausweis mit sich.

Somit besteht weiterhin das Dilemma, dass im Krankenhaus der mutmaßliche Wille des Verstorbenen eruiert werden muss. Meist geschieht dies durch Befragung der Angehörigen, was eine zusätzliche Belastung sowohl für das Krankenhauspersonal als auch insbesondere für die Angehörigen darstellt. Ein Leitfaden für TxB stellt in diesem Zusammenhang der Artikel „Entscheidungshilfe bei erweitertem intensivmedizinischem Behandlungsbedarf auf dem Weg zur Organspende“ dar [11] sowie für medizini- sche Belange der "Guide to the quality and safety for organs for transplantation“" [12].

Daher wurde im Herbst 2018 erneut die Einführung der Widerspruchslösung auch in Deutschland angeregt. Nach kontroversen und teils emotional geführten Debatten im Parlament, den Medien und in der Öffentlichkeit im Allgemeinen entschieden sich die Abgeordneten im Januar 2020 mehrheitlich für den alternativen Gesetzentwurf zur Stärkung der Entscheidungsbereitschaft und damit gegen eine Widerspruchslösung.

Vieles wird davon abhängen, ob die zuvor beschriebenen Maßnahmen des Gesetzes zur Stärkung der Entscheidungsbereitschaft tatsächlich greifen und der Schritt von der grundsätzlich positiven Einstellung zur Organspende zu einer Zunahme des dokumentierten Patientenwillens tatsächlich erfolgt. Das sprunghaft gestiegene Interesse an Organspendeausweisen und Infomaterialien im Rahmen der Gesetzesdebatte ist ein erstes positives Signal.

\section{I) Ziel ist, die Selbstbestim- mung bei Entscheidungen zur Behandlung am Lebensende frühzeitig zu fördern}

Verschiedene Maßnahmen zur Förderung der Information der Bürger sind im Gesetz vorgesehen. Ziel ist dabei, die individuelle Selbstbestimmung bei Entscheidungen zur Behandlung am Lebensende frühzeitig zu fördern. Dies geht über die reine Frage der Einstellung zur Organspende hinaus, sodass sich vielfältige Überlappungen zu Patientenverfügung, Vorsorgevollmacht und ggf. Palliativpass ergeben. Zur Aufklärung und Unterstützung in diesem komplexen Umfeld werden die Hausärzte vermehrt eingebunden. Sie sollen alle 2 Jahre über Organspende ergebnisoffen beraten und diese Beratung auch abrechnen können. Damit diese Beratung jeweils auf dem neuesten medizinischen Stand erfolgt, sind niederschwellige, wiederholte Fortbildungsangebote für die Hausärzte von zentraler Bedeutung. Die Landesärztekammern planen hierzu
Schulungsangebote, die durch OnlineAngebote ergänzt und vertieft werden. So hat die DSO ein spezielles E-LearningProgramm für Hausärzte entwickelt.

Des Weiteren sieht das Gesetz die Einrichtung eines Organspenderegisters zur Dokumentation der Entscheidung vor. Die entsprechenden Informationen im Register werden nach der Todesfeststellung für speziell berechtigte Ärzte zugänglich sein. Diese Beschränkung auf die Einsicht erst nach der Todesfeststellung stellt allerdings eine nicht unerhebliche Einschränkung in der intensivmedizinischen Praxis dar. Die Deutsche Interdisziplinäre Vereinigung für Intensivund Notfallmedizin e. V. (DIVI) ebenso wie die DSO haben darauf hingewiesen, dass die Einsichtnahme in das geplante Register bereits dann möglich sein sollte, wenn der irreversible Hirnfunktionsausfall bevorsteht oder als bereits eingetreten vermutet werden kann. Zu diesem Zeitpunkt ist die Entscheidung zur Fortführung intensivmedizinischer Maßnahmen einerseits oder zur Therapiebegrenzung andererseits zu treffen. Ähnlich wie der Zugriff auf die Patientenverfügung sollte in dieser Situation auch die Information im Organspenderegister zugänglich sein, um die Patientenautonomie sicherzustellen und zu gewährleisten, dass ein explizit geäußerter Wille zur Organspende nicht durch eine frühe Therapielimitierung ausgeschlossen wird.

\section{Bisherige Auswirkungen auf die Organspendezahlen}

Im vergangenen Jahr haben in Deutschland 932 Menschen nach ihrem Tod ein oder mehrere Organe für eine Transplantation gespendet [6]. Damit hat sich die Zahl der Organspender annähernd auf dem Niveau von 2018 gehalten. Es war auch nicht zu erwarten, dass sich die im letzten April angestoßenen Strukturmaßnahmen innerhalb weniger Monate in die Praxis umsetzen lassen. Was die organspendebezogenen Kontaktaufnahmen der Krankenhäuser zur DSO als Koordinierungsstelle betrifft, gibt es bereits eine positive Entwicklung: Die Meldungen von möglichen Organspendern stiegen im letzten Jahr um $7 \%$ auf 3020. 
Trotz COVID-19-Pandemie hat sich der positive Trend in den ersten 6 Monaten dieses Jahres fortgesetzt: Im Vergleich zum Vorjahreszeitraum gab es 7,3 \% mehr postmortale Organspender (2020: 487, 2019: 454) [13].

\section{Ausblick}

Es wird sicher auch weiterhin noch einige Zeit dauern, bis die neuen gesetzlichen Maßnahmen und der sie begleitende Initiativplan ihre volle Wirkung entfalten. Zusammen bieten sie die Chance, die Organspende in Deutschland zu fördern und damit mehr Menschen auf den Wartelisten mit einer lebensrettenden Transplantation zu helfen.

\section{Fazit für die Praxis}

\section{- Damit in der neu strukturierten Rechtslage eine Steigerung der Or- ganspende gelingt, ist eine strikte Umsetzung des Gesetzes vom letzten April in den Entnahmekrankenhäu- sern erforderlich. \\ - Die Transplantationsbeauftragten müssen ihre Rolle nach innen und außen leben, ebenso schulen und fortbilden. \\ - Die im Initiativplan vorgeschlagenen Maßnahmen müssen mit kurz-, mittel- und langfristigen Zielen weiter umgesetzt werden. \\ - Letztlich kann so auch in Deutschland eine „Kultur der Organspende" entstehen, wie sie bereits in anderen Ländern existiert.}

\section{Korrespondenzadresse}

\section{Prof. Dr. F. Braun, FEBS, MBA}

Sektion Klinische Transplantation, Klinik für Allgemeine, Viszeral-, Thorax-, Transplantations- und Kinderchirurgie, Universitätsklinikum Schleswig-Holstein (UKSH), Campus Kiel

Arnold-Heller-Str. 3 (Haus C), 24105 Kiel, Deutschland

felix.braun@uksh.de

\section{Einhaltung ethischer Richtlinien}

Interessenkonflikt. F. Braun und A. Rahmel geben an, dass kein Interessenkonflikt besteht.

Für diesen Beitrag wurden von den Autoren keine Studien an Menschen oder Tieren durchgeführt. Für die aufgeführten Studien gelten die jeweils dort angegebenen ethischen Richtlinien.

\section{Literatur}

1. Gesetze im Internet (1997) Gesetz über die Spende, Entnahme und Übertragung von Organenund Geweben (Transplantationsgesetz - TPG). https://www.organspende-info. de/fileadmin/Organspende/01_Informieren/ 06_Gesetze_und_Richtlinien/03_Gesetze_und Richtlinien/Transplantationsgesetz_TPG_.pdf. Zugegriffen: 15. Juni 2020

2. Deutsches Krankenhausinstitut e.V. (2012) Inhousekoordination bei Organspenden Abschlussbericht. https://www.dki.de/sites/ default/files/2019-01/inhousekoordination_bei organspenden.pdf.Zugegriffen: 15 . Juni 2020

3. Schulte K et al (2018) Rückgang der Organspenden in Deutschland. Dtsch Arztebl 115(27-28):463-468

4. Brauer M et al (2019) Wie viele potenzielle Organspender gibt es wirklich? Retrospektive Analyse zu nichterfolgter Diagnostik des irreversiblen Hirnfunktionsausfalls bei verstorbenen Patienten mit relevanter Hirnschädigung. Anaesthesist 68(1):22-29

5. Bundeszentrale für gesundheitliche Aufklärung (BZgA) (2020) Wissen, Einstellung und Verhalten zur Organ- und Gewebespende. https:// www.organspende-info.de/zahlen-und-fakten/ einstellungen-und-wissen.html. Zugegriffen: 15 Juni 2020

6. Deutsche Stiftung Organtransplantation (DSO) (2020) DSO Jahresbericht. https://www.dso.de/ organspende/statistiken-berichte/jahresbericht Zugegriffen: 15. Juni 2020

7. Bundesgesetzblatt im Internet (2019) Zweites Gesetz zur Änderung des Transplantationsgesetzes - Verbesserung der Zusammenarbeit und der Strukturen bei der Organspende. https://www.bundesgesundheitsministerium.de/ fileadmin/Dateien/3 Downloads/Gesetze und Verordnungen/GuV/O/GZSO_BGBI.pdf. Zugegriffen: 15 . Juni 2020

8. Bundesministerium für Gesundheit (2019) Gemeinschaftlicher Initiativplan Organspende. https://www.bundesgesundheitsministerium. de/fileadmin/Dateien/3_Downloads/O/ Organspende/Initiativplan_Organspende. pdf. Zugegriffen: 15. Juni 2020

9. Bundesgesetzblatt im Internet (2020) Gesetz zur Stärkung der Entscheidungsbereitschaft bei der Organspende. https://www.bgbl.de/xaver/ bgbl/start.xav?startbk=Bundesanzeiger_BGBI\& jumpTo=bgbl120s0497.pdf\#_bgbl_\%2F\%2F* \%5B\%40attr id\%3D\%27bgbl120s0497.pdf\%27 \%5D_1591989775189.Zugegriffen: 15.Juni 2020

10. Küpers M et al (2020) Organspendeausweise bei Schockraumpatienten. Dtsch Arztebl 117:183-187

11. Neitzke $G$ et al (2019) Entscheidungshilfe be erweitertem intensivmedizinischem Behandlungsbedarf auf dem Weg zur Organspende. Med Klin Intensivmed Notfmed 114:319-326
12. EDQM Council of Europe (2020) NEW release: 7th edition guide to the quality and safety of organs for transplantation. https://www.edqm.eu/en/ news/new-release-7th-edition-guide-qualityand-safety-organs-transplantation. Zugegriffen: 15. Juni 2020

13. Deutsche Stiftung Organtransplantation (DSO) (2020) Statistiken zur Organspende im Überblick. https://www.dso.de/organspende/statistikenberichte/organspende. Zugegriffen: 15. Juli 2020 